\title{
Mycoplasmes de la chèvre apparentés à l'espèce Mycoplasma dispar
}

\author{
par P. PERREAU (*) \\ (avec la collaboration technique de A. BREARD, C. BRIERE et C. LEGOFF)
}

\section{RESUME}

\begin{abstract}
Quatre souches de mycoplasmes isolées chez la chèvre à partir de lésions de pneumonie enzootique et de bronchite vermineuse, d'origine géographique très différente (Ethiopie, France, Tchad), se révèlent appartenir à une même espèce, très voisine de $M$. dispar (Gourlay et Leach, 1970).

Cette conclusion découle d'une étude comparative portant sur leurs caractères culturaux, métaboliques et antigéniques. Les méthodes sérologiques sont la séro-agglutination, l'immunofluorescence, la précipitodiffusion en milieu gélifié, l'inhibition de croissance; l'analyse antigénique est complétée par l'électrophorèse en gel de polyacrylamide.
\end{abstract}

En dehors des mycoplasmoses essentielles que sont la pleuropneumonie et l'agalaxie contagieuses de la chèvre, dues respectivement à $M$. mycoides var. capri et $M$. agalactiae, de multiples observations d'infections non spécifiques à mycoplasmes enrichissent de jour en jour nos connaissances sur la pathologie caprine.

Des synthèses récentes ont tenté de mettre à jour ce dossier complexe [COTTEW (3), COTTEW et LEACH (4), HUDSON et collab. (7), MEYER (9)].

Dans les syndromes infectieux respiratoires des petits ruminants, et singulièrement dans la pneumonie enzootique de la chèvre, on sait que postérieurement ou simultanément à des infections virales ou bactériennes des mycoplasmes interviennent, dont le pouvoir patho-

(*) Institut d'Elevage et de Médecine vétérinaire des Pays tropicaux, 10, rue Pierre Curie, 94700 Maisons-Alfort. gène reste encore à définir, en admettant même qu'il existe.

$M$. mycoides var. mycoides, agent spécifique de la péripneumonie contagieuse des bovidés, a été déjà isolé de lésions de pneumonie enzootique de la chèvre ou de pleuropneumonie contagieuse non distincte sur le plan anatomoclinique de l'infection spécifique à $M$. capri [HUDSON et collab. (7); PERREAU (10)].

Un autre mycoplasme fréquemment rencontré dans le tractus respiratoire des petits ruminants est $M$. arginini, dont l'ubiquité est manifeste tant par le nombre d'espèces qu'il contamine que par la diversité des lésions à partir desquelles on l'isole [LEACH (8)].

Les observations que nous rapportons ici concernent quatre souches de mycoplasmes isolées de l'appareil respiratoire de chèvres atteintes de pneumonie enzootique dont les caractères culturaux et antigéniques sont identiques, ce qui nous autorise à les considérer comme appartenant à une même espèce, très voisine de $M$. dispar comme on le verra. 


\section{MATERIEL ET METHODES}

\section{A. Souches à déterminer}

1. Souches $\mathrm{C}_{2}$ et $\mathrm{C}_{3}$ :

D'origine éthiopienne, elles furent isolées à partir de prélèvements effectués chez des chèvres atteintes d'une pneumonie contagieuse déclenchée par la mise en stabulation. Il s'agissait d'animaux achetés et groupés au laboratoire de Debré-Zeit pour la production du vaccin capripestique, c'est-à-dire de conditions tout à fait semblables à celles que nous avons eu l'occasion de décrire pour un autre foyer de pneumonie contagieuse (11).

\section{Souche $\mathbf{C}_{13}$ :}

Originaire du Tchad, elle fut isolée d'un prélèvement effectué au laboratoire de Farcha à Fort-Lamy. Là aussi, une pneumonie contagieuse, parfois accompagnée de pleurésie, sévissait sur des chèvres autochtones.

Pour ces trois premières souches, les prélèvements reçus consistaient en de petits blocs de tissu pulmonaire hépatisé, soigneusement découpés dans des lésions aiguës, introduits dans des flacons à pénicilline et lyophilisés sans addition aucune d'antibiotiques.

La méthode s'est révélée excellente, surtout si les flacons ont été ensuite bouchés sous vide et conservés à $-25^{\circ} \mathrm{C}$; clle a permis une analyse microbiologique précise sans aucun impératif de temps; certains prélèvements ont été étudiés plusieurs mois après leur réception.

\section{Souche $\mathrm{C}_{14}$ :}

Cette quatrième souche, d'origine métropolitaine, nous a été confiée par nos confrères de l'Institut Pasteur de Paris, sous forme d'une primo-culture sur milieu gélosé ensemencé par le mucus trachéal d'une chèvre atteinte de bronchite vermineuse à Mullerius capillaris.

\section{B. Souches de référence}

Ce sont des souches reçues de Centres officiels ou isolées dans notre laboratoire et conservées dans la collection de I'I.E.M.V.T.

L'étude comparative de base a utilisé les espèces suivantes :

M. mycoides var. mycoides: $\mathbf{B}_{17}$ et Sankhaye; M. mycoides var. capri : $\mathrm{Pg}_{3}, \mathrm{OSB}_{42}, \mathrm{IPX}$;
M. agalactiae : $\mathbf{P g}_{2}$;

M. arginini: G 230 et T.O.;

$M$. dispar: 10125 NCTC.

\section{Milieux de culture et métabolisme}

Nous n'insisterons pas sur la composition des milieux très classiques employés pour la culture des souches de collection précitées.

Toutefois, signalons que la souche 10125 NCTC de $M$. dispar cultive de façon satisfaisante sur le milieu de Hayflick contenant de l'extrait de levure préparé selon le procédé de HERS (6). Aussi avons-nous délaissé le milieu décrit initialement par GOURLAY et LEACH (5).

$\mathrm{Au}$ départ, les souches caprines étudiées ici n'étaient pas d'un entretien facile et les premières subcultures ont été assez aveugles. Après une adaptation progressive, deux milieux liquides nous ont permis de préparer des antigènes en quantité suffisante pour les tests sérologiques et la préparation des sérums :

1. Le milieu de Hayflick dont nous venons de parler;

2. Un milieu au tryptose (TSp) employé couramment dans notre laboratoire pour tous les mycoplasmes à culture malaisée. Sa composition est la suivante pour un $\mathrm{pH}$ de 7,6 :

Bacto-Tryptose Difco . . . 20

Bacto-Yeast-Extract Difco . . $5 \mathrm{~g}$

Glucose . . . . . . $2 \mathrm{~g}$

Phosphate disodique anhydre . $\quad 2,5 \mathrm{~g}$

Chlorure de sodium . . . $5 \mathrm{~g}$

Extrait frais de levure (Hers) . $25 \mathrm{ml}$

Digestion papaïnique de cœur de bouf . . . . $50 \mathrm{ml}$

Sérum de cheval . . . . $200 \mathrm{ml}$

Eau distillée Q.S. pour . . $1.000 \mathrm{ml}$

Leur gélification par une très faible quantité de «Ionagar» OXOID (à 0,02 p. 100 en concentration finale) permit d'entretenir les souches par culture en profondeur en bonnes conditions de microaérobiose.

Les milieux solides correspondants étaient obtenus par adjonction de $10 \mathrm{~g}$ de «Ionagar» par litre. 
La recherche des caractères biochimiques de ces souches fut effectuée selon les procêdés décrits par ALUOTTO et collab. (1) et surtout selon ceux préconisés par le Centre de Référence pour les mycoplasmes animaux d'Aarhus (Directeur: Professeur E.A. FREUNDT) qui mettent en cuvre le milieu de base suivant:

Heart Infusion Broth Difco $180 \mathrm{ml}$

Sérum de cheval . . . $40 \mathrm{ml}$

Extrait frais de levure . . $20 \mathrm{ml}$

A.D.N. (solution à 0,2 p.

100). . . . . $2,6 \mathrm{ml}$

Rouge de phénol (à $1 \mathrm{p}$. 1.000)

$5 \quad \mathbf{m l}$

Pénicilline . 100.000 U.I.

Acétate de thallium (à 1/80)

$2,5 \mathrm{ml}$

Selon l'activité biochimique à rechercher, on ajoute à cette composition l'un des substrats suivants :

Glucose

$1 \mathrm{~g}$

Arginine (chlorhydrate) .

$2,5 \mathrm{~g}$

Urée

$2 \mathrm{~g}$

Triphenyltetrazolium (chloru-

re) à 2 p. 100

$5 \mathrm{ml}$

Le $\mathrm{pH}$ final est ajusté à 7,8 pour l'hydrolyse du glucose, à 7,3 pour l'hydrolyse de l'arginine et celle de l'urée, à 7,5 pour la réduction des sels de tétrazolium.

La lecture de ces tests était effectuée au bout de 8 à 10 jours de culture et le $\mathrm{pH}$ terminal mesuré au moyen d'un pH-mètre Methrom E 396.

Les épreuves d'hémolyse ont été faites sur milieu gélosé contenant 0,5 p. 100 d'hématies de mouton; après ensemencement copieux en stries, la lecture était faite le $5^{t}$ jour en lumière transmise, au moyen d'un stéréomicroscope.

La recherche d'une hémagglutinine fut effectuée avec des suspensions d'hématies de mouton et de lapin à 0,5 p. 100 selon un protocole classique de titrage. Des cultures de 4 jours et des suspensions de germes lavés et lyophilisés ( $5 \mathrm{mg} / \mathrm{ml}$ ) étaient dilués du $1 / 2$ au $1 / 1024$; chaque dilution recevait à volume égal la suspension d'hématies.

\section{Examens microscopiques}

A l'état frais en contraste de phase; après coloration au May-Grunwald-Giemsa, en immunofluorescence.

\section{E. Inoculation aux embryons de poule}

Par la voie intravitelline, à des œufs embryonnés de 6 jours, aux doses de $0,1-0,2$ $0,3 \mathrm{ml}$ de culture de 3 jours pour chaque souche.

\section{F. Antigènes}

Trois types d'antigènes furent systématiquement préparés :

1. Une suspension de mycoplasmes lavés par centrifugation, en solution physiologique formolée à 3 p. 100 et ajustée à l'opacité du tube $n^{6} 2,5$ de Brown, pour la séro-agglutination.

2. Un antigène total obtenu par traitement aux ultra-sons d'une suspension dense de germes (10) pour la précipito-diffusion en gel.

3. Des germes lyophilisés, débarrassés de toute trace du milieu de culture, pour l'électrophorèse en gel de polyacrylamide.

\section{G. Sérums}

Les immunsérums employés furent préparés sur lapin ou sur mouton selon un protocole déjà décrit (11).

\section{H. Protocoles techniques}

1. Séro-agglutination: c'est une méthode lente en tubes. A une série de dilutions de sérum allant du $1 / 10$ au $1 / 5120$, on ajoute un volume égal de la suspension normalisée de mycoplasmes.

Après agitation et séjour de 18 heures à $37^{\circ} \mathrm{C}$, la lecture est effectuée à l'aide d'un stéréomicroscope; le titre correspond à la plus haute dilution donnant des agglutinats nets.

2. Précipito-diffusion en gel : méthode tout à fait banale, en boîtes de Pétri contenant le milieu suivant à $\mathrm{pH} \mathrm{7,2}$ :

$\begin{array}{lllll}\text { Bacto-Agar Noble Difco . } & . & 12 & \mathrm{~g} \\ \text { Merthiolate de sodium } & . & . & 0,20 & \mathrm{~g} \\ \text { P.B.S. (Dulbecco), Q.S. } & . & . & 1.000 & \mathrm{ml}\end{array}$ 
3. Immunofluorescence : selon des principes déjà décrits (11). Seule la méthode indirecte a servi; les conjugués fluorescents anti-lapin et anti-mouton sont ceux que commercialise l'Institut Pasteur de Paris.

Les préparations étaient des films de culture de 2 à 3 jours et des empreintes de colonies fixées au Bouin.

4. Inhibition de croissance: sur milieu solide, par la méthode dite des disques, selon un procédé analogue à celui de CLYDE (2), à quelques variations près, portant sur le diamètre des disques et la quantité de sérum utilisée (disques B-D Mérieux, d'un diamètre de $5,5 \mathrm{~mm}$, saturés par $0,05 \mathrm{ml}$ de sérum pur).

5. Electrophorèse comparative en gel de polyacrylamide : effectuée en suivant à la lettre le protocole mis au point par RAZIN et ROTTEM (13).

Nous avons employé le système analytique Acrylophor Pleuger (mod. 146) qui permet 8 électrophorèses simultanées dans des tubes de $6 \mathrm{~mm}$ de diamètre sur 65 de hauteur.

Chaque gel reçoit à sa partie supérieure d'abord $0,15 \mathrm{ml}$ d'antigène préparé selon la méthode originale (lyse des germes lyophilisés par le mélange phénol/acide acétique/eau dans les proportions $2 / 1 / 0,5$ p. v.v.), puis $0,05 \mathrm{ml}$ d'une solution de saccharose à 40 p. 100 dans de l'acide acétique à 50 p. 100 .

L'électrophorèse est en principe achevée au bout de 3 heures environ, avec une tension de $5 \mathrm{~mA}$ par tube.

Le colorant «marqueur » est la rhodamine B en solution à 0,5 p. 100 ; les gels sont colorés au Noir-Amide $10 \mathrm{~B}$ (Merck) à 0,6 p. 100 durant 30 minutes, puis décolorés soit par électrophorèse soit par simples lavages dans un bain d'acide acétique à 7 p. 100 .

\section{RESULTATS}

\section{Cultures}

A l'isolement, la culture de ces souches est toujours discrète.

En milieu liquide, un trouble léger apparaît en 4 à 5 jours et le transfert en milieu neuf doit se faire dans les 2 à 3 jours qui suivent. On observe ensuite la sédimentation de fins granules qui ne sont que des micro-colonies en suspension.

Sur milieu gélosé, les colonies apparaissent dans les mêmes délais. D'un diamètre variant de 80 à $300 \mu$, elles sont caractérisées par leur aspect très atypique par rapport à la morphologie classique des colonies de mycoplasmes.

Translucides, et de ce fait peu visibles au départ, de taille et de contour très irréguliers, sans noyau central dense, elles ne sont décelables qu'avec l'aide du stéréomicroscope et en lumière transmise obliquement. Elles ressemblent à de fines gouttes de mucus dont la surface se couvre bientôt d'un fin réseau brillant et en relief (voir les photos $n^{\circ} 1,2$ et 3 ); très friables, elles se fragmentent dès qu'on les touche.

L'entretien de ces souches est assez difficile et seuls les passages en milieu liquide nous ont permis de les adapter à la croissance en milieu artificiel; au début, les transferts de gélose à gélose n'ont pas été suivis de succès.

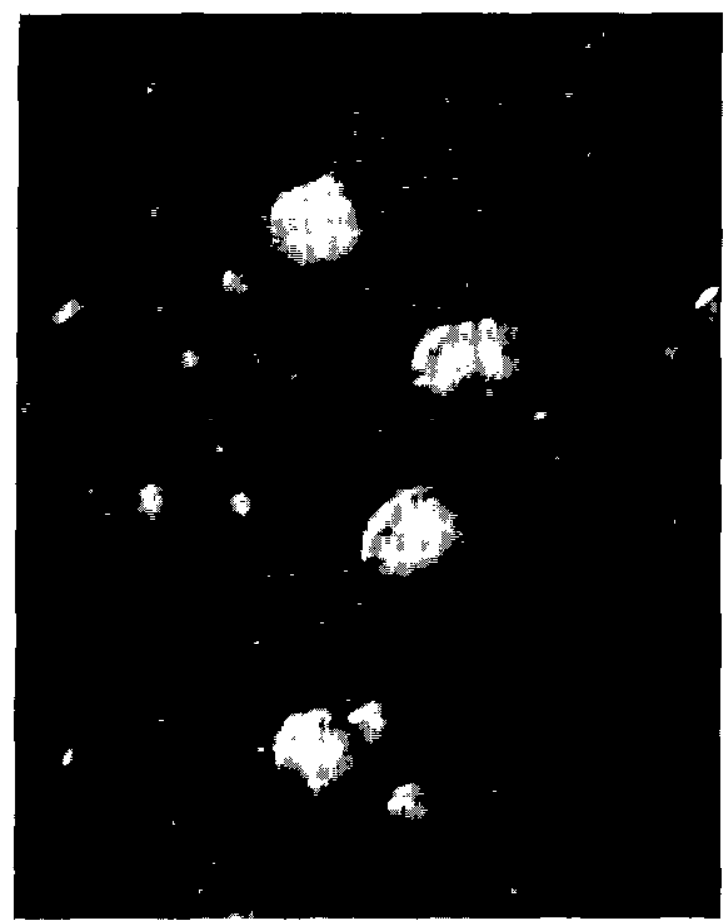

Photo 1. - Souche $\mathrm{C}_{2}$ : colonies en lumière incidente. Grossissement : 50. 


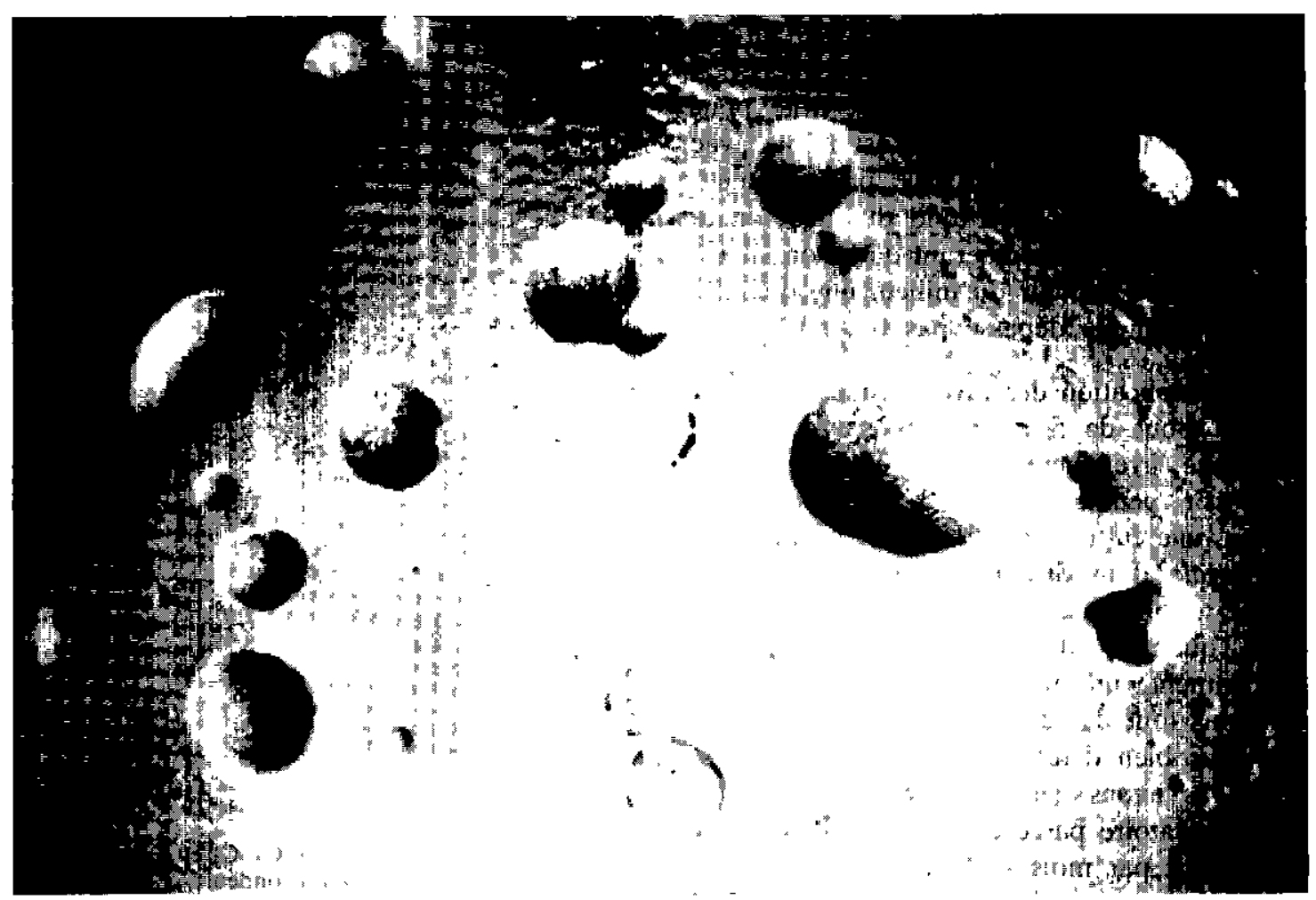

Photo 2. - Souche $C_{4}$ : colonies en lumière transmise oblique. Grossissement : 75 .

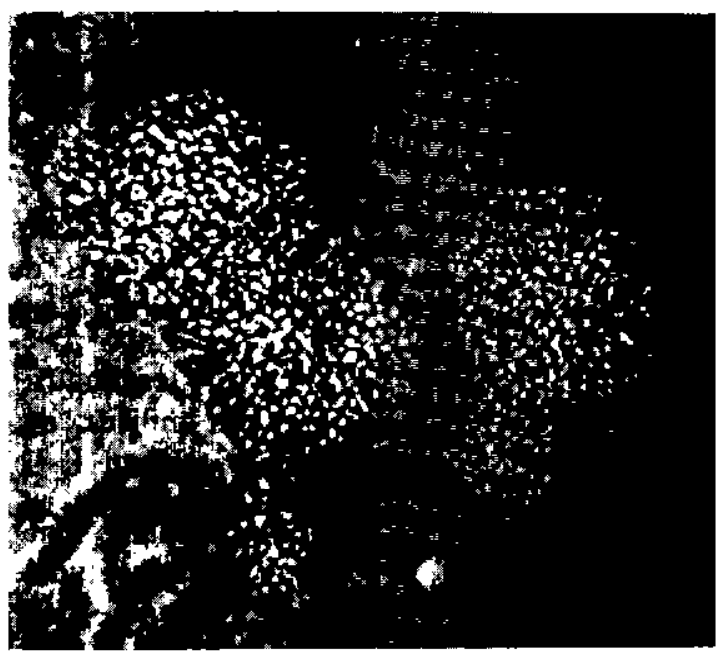

Photo 3. - Souche $\mathrm{C}_{13}$ : colonies confluentes. Coloration de Dienes. Grossissement: 300. L'aspect réticulć de ces colonies sans noyau central dense est tout à fait conforme à la description originale de $M$. dispar. 
L'ensemencement de ces premières cultures par piqûre profonde en milieu de Hayflick faiblement gélosé s'est révélé fort utile pour leur conservation; l'inoculation est faible avec une pipette Pasteur très fine, préalablement remplie, puis plongée au fond du tube; on la remonte lentement, en libérant dans son sillage et sur toute la hauteur du milieu, une quantité de culture qui ne dépasse pas $0,2 \mathrm{ml}$.

La multiplication des mycoplasmes se manifeste au bout de 5 à 6 jours par une strie réfringente, irrégulière, discrète mais nette, qui va grossir pendant 2 ou 3 semaines; bien souvent, une culture en surface apparaît ensuite sous forme d'un disque très mince, d'aspect pailleté, appliqué à la surface du gel sous la petite quantité de liquide qui surmonte constamment un tel milieu (photo $n^{\circ} 4$ ). Indiquons tout de suite que ce type de culture vaut pour bien d'autres espèces de mycoplasmes; nous avons généralisé son emploi dans notre laboratoire parce qu'il permet d'effectuer un transfert par mois seulement, de gel à gel, sans risque de perdre des souches dont la culture par les procédés classiques est au même moment d'un succès très aléatoire.

En tout cas, les conditions de la microaérobiose ainsi réalisée semblent excellentes; l'incubation de boîtes de Pétri classiques dans un mélange d'azote $(95$ p. 100$)$ et d'anhydride carbonique ( 5 p. 100) nous a donné de moins bons résultats.

Après une adaptation qui n'a demandé qu'une série de 7 à 8 passages sur ces milieux, il est apparu que le milieu de Hayflick gélosé était supérieur au milieu TSp solide; les colonies y sont de plus grande taille et bien visibles dans un court délai ( 3 jours au lieu de 5 en moyenne pour le milieu TSp).

$\mathrm{Au}$ contraire, en phase liquide, l'ordre de leur valeur est renversé; le milieu TSp nous a permis de préparer les antigènes destinés aux tests sérologiques, avec d'excellentes cultures obtenues en 3 jours, d'une densité nettement plus élevée que sur milieu de Hayflick.

La souche 10125 NCTC de $M$. dispar s'est comportée d'une façon absolument identique sur les mêmes milieux; ses colonies, atypiques aussi, étaient tout à fait semblables à celles des souches caprines étudiées ici.

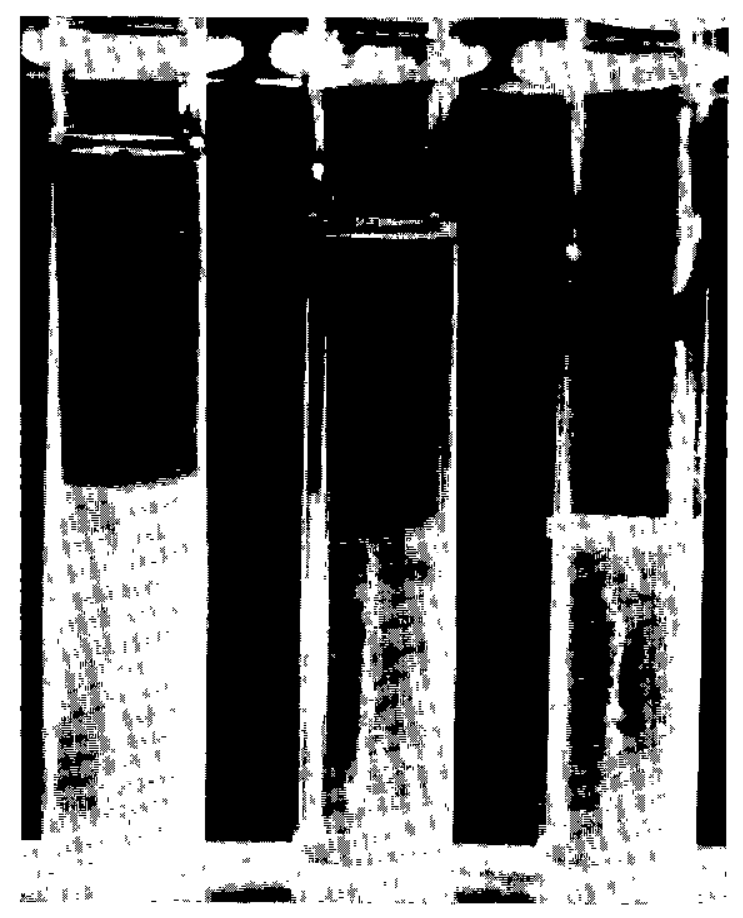

Photo 4. - Souches $\mathrm{C}_{2}, \mathrm{C}_{s}$ et $\mathrm{C}_{13}$. Culture en profondeur.

A l'examen microscopique, on retrouve à l'évidence le polymorphisme habituel des mycoplasmes; ici encore aucune différence n'a pu être décelée entre ces souches et $M$. dispar.

Un caractère commun a été cependant noté; dans les cultures jeunes, des formes filamenteuses existent en proportion notable, qui évoquent irrésistiblement celles que les souches sauvages de $M$. mycoides montrent en début de culture.

\section{Métabolisme}

Toutes ces souches fermentent le glucose et réduisent les sels de tétrazolium; mais elles n'hydrolysent ni l'arginine, ni l'urée.

Les $\mathrm{pH}$ terminaux, après 10 jours de culture, dans les milieux de l'A.M.R.C. sont relevés dans le tableau $\mathbf{I}$.

Ces résultats sont très homogènes pour les cinq souches étudiées, à l'exception du $\mathrm{pH}$ final : 7,45 noté pour le milieu à l'urée contenant la souche $C_{14}$. Ce test a été renouvelé plusieurs fois avec un résultat identique et non expliqué. L'abaissement du $\mathrm{pH}$ dans le milieu au glucose est très significatif. 


\begin{tabular}{|c|c|c|c|c|}
\hline \multirow[b]{2}{*}{ Souches : } & \multicolumn{4}{|c|}{ Substrats des milieux d'épreuve et $\mathrm{pH}$ initiaux : } \\
\hline & $\begin{array}{c}\text { Arginine } \\
7,3\end{array}$ & $\begin{array}{c}\text { Glucose } \\
7,8\end{array}$ & $\begin{array}{r}\text { Urëe } \\
7,3\end{array}$ & $\begin{array}{c}\text { Tëtrazolium } \\
7,5\end{array}$ \\
\hline $\mathrm{c}_{2}$ & 6,70 & 6,30 & 6,95 & + \\
\hline $\mathrm{C}_{3}$ & 6,70 & 6,55 & 6,90 & + \\
\hline$c_{13}$ & 6,90 & 6,40 & 6,85 & + \\
\hline$c_{14}$ & 6,95 & 6,40 & 7,45 & + \\
\hline $\begin{array}{l}10125 \\
\text { (M. dispar) }\end{array}$ & 7,10 & 6,55 & 6,95 & + \\
\hline
\end{tabular}

La recherche d'une hémolyse dans nos conditions expérimentales a toujours été négative.

Mais toutes ces souches agglutinent les hématies de lapin et de mouton. Le phénomène est particulièrement net avec les germes lyophilisés remis en suspension $(5 \mathrm{mg} / \mathrm{ml})$ et les globules rouges de lapin; les titres atteignent $1 / 256$ et $1 / 514$.

\section{Pouvoir pathogène sur l'embryon de poulet}

Il semble nul car aucun embryon n'est mort dans les jours qui ont suivi l'inoculation intravitelline aux doses déjà indiquées.

Une forte proportion des poussins n'a pu percer la coquille le jour de l'éclosion; toutefois tous ceux qui ont éclos n'avaient aucune malformation ou lésion particulière.

\section{Tests sérologiques}

a) Séro-agglutination : les résultats sont consignés dans le tableau II. Très clairs, ils révèlent une communauté antigénique importante entre ces souches caprines et la souche $\mathrm{n}^{\mathrm{0}} 10125$.

On note bien quelques réactions croisées à bas titre avec d'autres espèces communes chez la chèvre, notamment $M$. agalactiae, $M$. arginini et $M$. capri; cela n'est pas pour surprendre, mais en tout état de cause leur titre est sans commune mesure avec celui des agglutinations croisées avec $M$. dispar.

b) Immunofluorescence : ils confirment parfaitement les résultats précédents et sont notés dans le tableau III.

c) Précipito-diffusion en gel : les photos $\mathrm{n}^{\text {o8 }} 5$ et 6 et leurs schémas correspondants en montrent nettement les résultats.

On peut voir que les quatre souches caprines $\mathrm{C}_{2}, \mathrm{C}_{3}, \mathrm{C}_{13}$ et $\mathrm{C}_{14}$ ont une constitution antigénique identique et qu'elles sont réellement à considérer comme représentatives d'une même espèce, ce qui sera vérifié plus loin par l'analyse électrophorétique.

En effet, six lignes communes de précipitation apparaissent entre le réservoir de sérum précipitant et les antigènes totaux des quatre souches caprines $\left(\mathrm{C}_{2}, \mathrm{C}_{3}, \mathrm{C}_{13}\right.$ et $\left.\mathrm{C}_{14}\right)$. Devant la cupule contenant l'antigène total de $M$. dispar, quatre seulement de ces lignes persistent.

Cette parenté sérologique est mise en évidence de la même façon lorsque cette série d'antigènes est confrontée à un sérum précipitant anti-M. dispar. Comme on le voit sur la photo $\mathrm{n}^{\circ} 6$, deux lignes seulement semblent spécifiques de cette souche; tout le reste est commun.

A noter que les sérums précipitants antiagalactiae, anti-arginini, anti-capri et antimycoides opposés à cette même série d'antigènes n'ont donné aucune ligne de précipitation. 
TABLEAU $N^{\circ} \mathrm{II}$

Rêsultats des tests de sêroagglutination

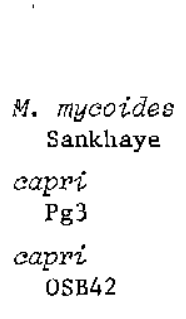

u eapri

$\rightarrow$ agalactiae

P Pg2 a arginini

$\rightarrow$ T. 0 .

dispar

F $\quad 10125$

$\mathrm{C}_{2}$

$\mathrm{c}_{3}$

$\mathrm{c}_{13}$

$c_{14}$

\begin{tabular}{|c|c|c|}
\hline $\begin{array}{c}\text { M. mycoides } \\
\text { Sankhaye }\end{array}$ & $\begin{array}{l}\text { capri } \\
\text { Eg3 }\end{array}$ & $\begin{array}{l}\text { copri } \\
\text { osB42 }\end{array}$ \\
\hline 2560 & & \\
\hline & $>5120$ & $\cdot$ \\
\hline
\end{tabular}

2560

$\begin{array}{lllll} & & & & 5120 \\ - & 80 & 40 & 80 & - \\ - & - & - & - & - \\ - & - & - & 20 & - \\ - & 40 & 20 & - & -\end{array}$

$$
\text { A n } t \text { i g è n e } s
$$

agalactiae arginini dispar

$\mathrm{C}_{2}$

\begin{tabular}{lll}
$\mathrm{c}_{3}$ & $\mathrm{c}_{13} \quad \mathrm{c}_{14}$ \\
\hline
\end{tabular}

-

-

-

$-$

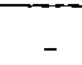

NB. Les nombres expriment 1 'inverse des dilutions de sërum. 


\begin{tabular}{|c|c|c|c|c|c|}
\hline Sérums & $\mathrm{C}_{2}$ & $c_{3}$ & $\begin{array}{l}\text { i g } \\
c_{13}\end{array}$ & $c_{14}$ & $\begin{array}{c}10125 \\
\text { (M. dispar) }\end{array}$ \\
\hline$c_{2} \quad 1 / 50$ & +++ & +++ & +++ & +++ & ++ \pm \\
\hline$c_{3} \quad 1 / 50$ & +++ & +++ & ++ & +++ & ++ \\
\hline$c_{13} 1 / 50$ & ++ & +++ & $t+t$ & +++ & ++ \pm \\
\hline $\mathrm{C}_{14} 1 / 50$ & +++ & +++ & $+t+$ & +++ & +++ \\
\hline $\begin{array}{l}\text { M. dispar } 10125 \\
1 / 50\end{array}$ & ++ \pm & ++ \pm & ++ \pm & ++ & +++ \\
\hline $\begin{array}{l}\text { M. agalactiae Pg2 } \\
1 / 50\end{array}$ & - & - & \pm & \pm & - \\
\hline $\begin{array}{l}\text { M. } \\
\text { arginini } \\
1 / 50\end{array}$ & - & - & - & - & - \\
\hline $\begin{array}{l}\text { M. capri Pg3 } \\
1 / 50\end{array}$ & \pm & \pm & - & - & - \\
\hline $\begin{array}{l}\text { M. myooides vom } \\
1 / 50\end{array}$ & - & - & - & - & - \\
\hline
\end{tabular}

d) Inhibition de croissance: les résultats n'ont pas été aussi clairs qu'on l'espérait, car nos sérums préparés sur lapin n'étaient pas fortement inhibiteurs pour les souches homologues.

Cependant, il fut possible de voir que les sérums anti- $C_{2}$, anti- $C_{3}$, anti- $C_{13}$ et anti- $C_{14}$ provoquaient une inhibition croisée au moins partielle pour ces souches et la souche 10125 .

L'inverse n'était pas vrai; le sérum antidispar 10125 ne semblait pas inhiber la croissance des souches caprines.

\section{Electrophorèse en gel de polyacrylamide}

Cette méthode permet, on le sait, la comparaison des structures protéiques de diverses espèces de mycoplasmes; elle s'est révélée féconde pour apparenter et grouper les souches.

L'identité de $\mathrm{C}_{2}, \mathrm{C}_{3}, \mathrm{C}_{13}$ et $\mathrm{C}_{14}$ devient ici d'une parfaite évidence comme le montre la photo $\mathrm{n}^{\mathrm{0}} 7$.

On remarque aussi que le schéma de structure protéique de $M$. dispar est très voisin mais non identique, car il n'y a pas concordance des fractions et des distances de migration, au moins dans la partie supérieure du gel.

Toutefois, il semble s'agir de diftérences mineures, surtout si on compare cette série d'électrophorégrammes avec ceux des autres mycoplasmes connus de la chèvre, qui ne permettent vraiment aucun apparentement.

Ajoutons que ce procédé nous a permis de séparer nettement les souches caprines étudiées de nombreuses espèces de mycoplasmes en dehors de celles que nous avons citées et au premier rang desquelles $M$. bovirhinis, $M$. agalactiae var. bovis, $M$. bovigenitalium et les mycoplasmes classés sous les rubriques provisoires actuelles de groupes 6,7 et 8 de LEACH (4). 

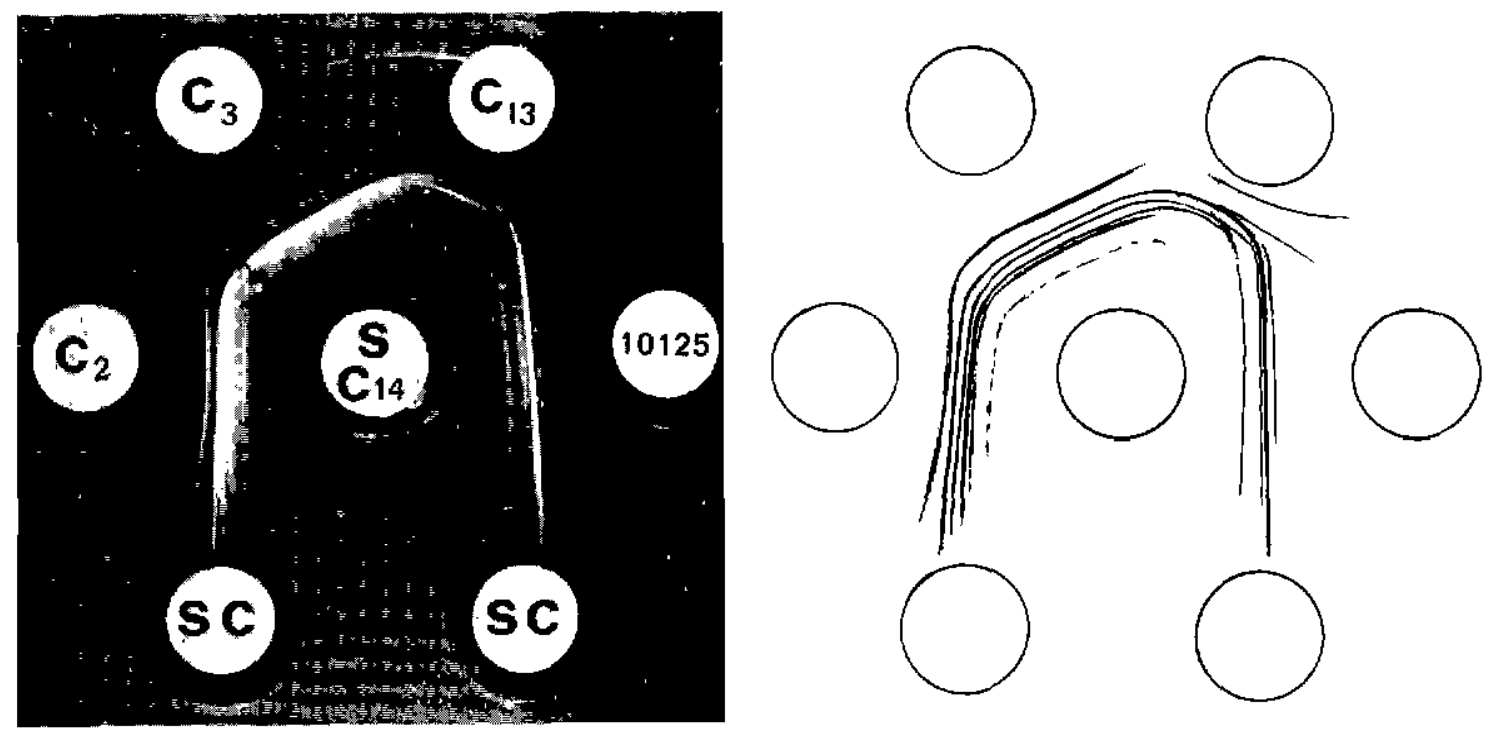

Photo 5. - Le sérum précipitant anti- $\mathrm{C}_{1+}$ est au centre; les deux cupules inférieures (SC) contiennent le sérum de cheval $1 / 50$ destiné à révéler d'éventuelles précipitines anti-sérum de cheval (constituant du milieu). L'antigène $\mathrm{C}_{1 \mathfrak{s}}$ est moins concentré que les autres, d'où j'éloignement et le flou relatif des lignes.
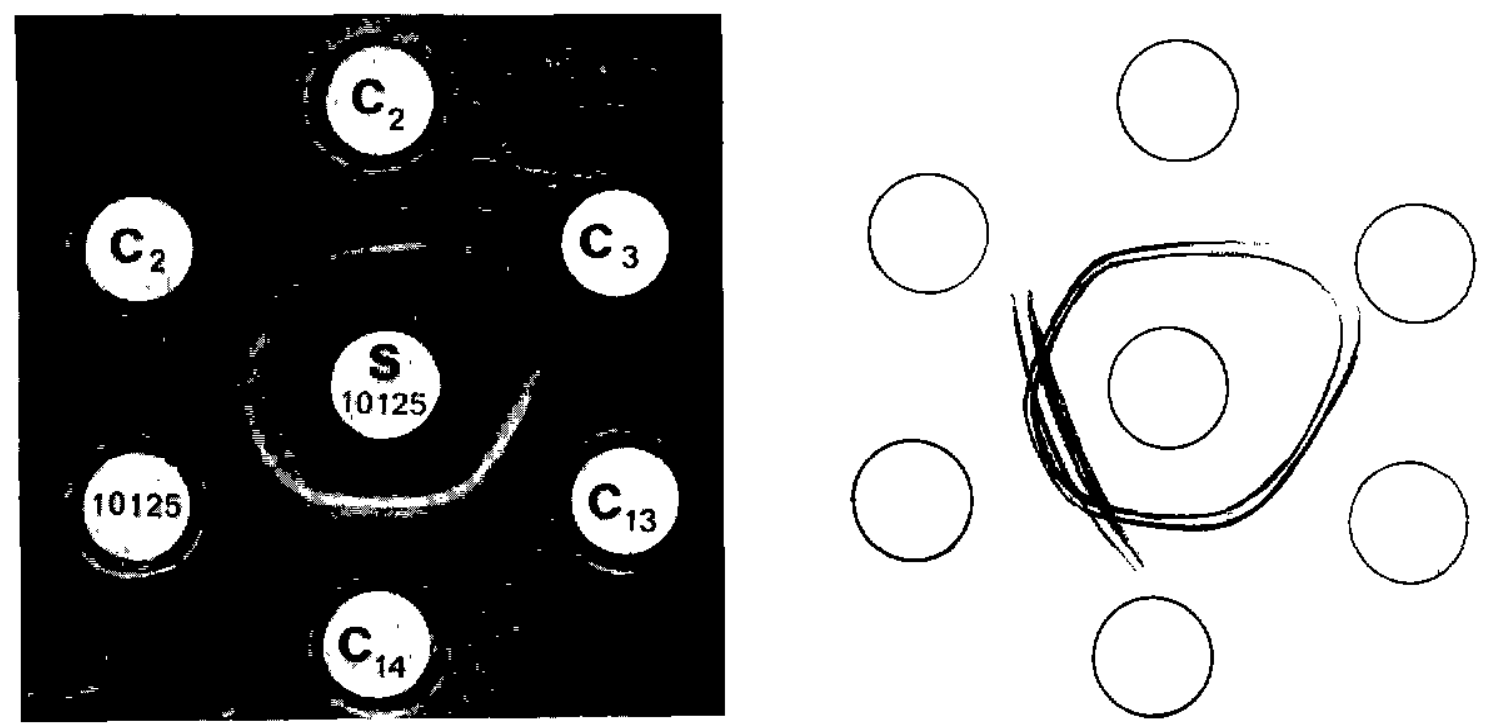

Photo 6. - Le sérum précipitant anti-dispar (10125) est au centre.

Ici c'est l'antigène $C_{3}$ qui est le moins concentré. 


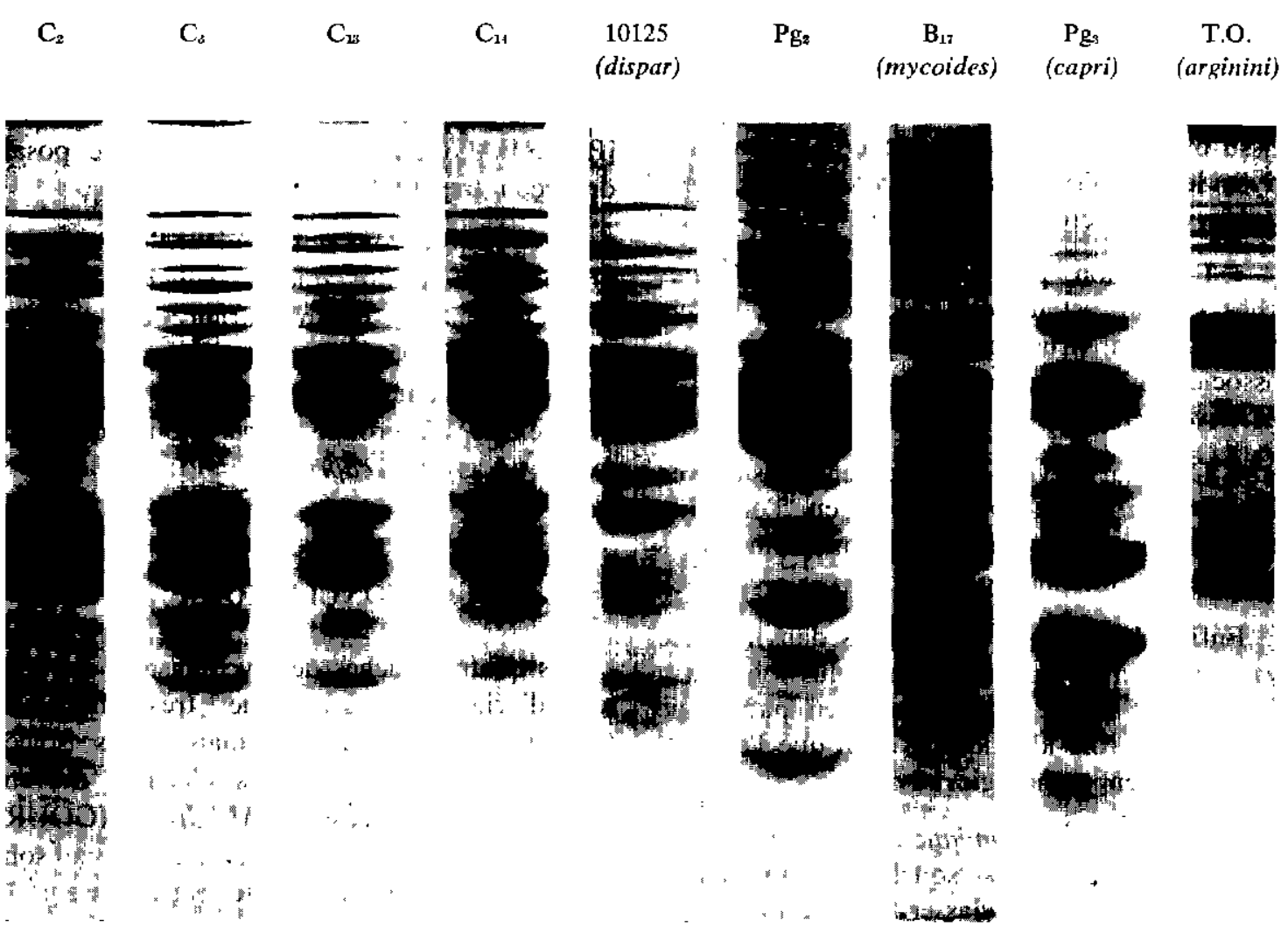

Photo 7. - Electrophorèse en gel de polyacrylamide: l'identité des souches $C_{2}, C_{3}, C_{1}$ et $\mathrm{C}_{4}$ est manifeste. On remarquera en passant les schémas identiques (ou presque) de $M$. mycoides var. mycoides et de $M$. mycoides var. capri.

\section{COMMENTAIRES}

Des commentaires de plusieurs ordres sont à faire :

1. D'ordre microbiologique: il faut souligner l'intérêt que présente pour le laboratoire de diagnostic la réception de fragments de lésion lyophilisés en bloc. C'est certainement grâce à ce type de prélèvement que nous avons pu isoler trois souches, des mois après l'autopsie des chèvres, sans contamination bactérienne autre que la flore associée dans les lésions. Il est bien certain qu'on ne peut généraliser cette méthode, mais c'est une possibilité dont il faudra se souvenir dans les laboratoires qui ne peuvent se consacrer à tout moment à de tels diagnostics, pour des raisons diverses (manque de personnel, de matériel, de milieux appropriés).

2. D'ordre pathologique: les souches $\mathrm{C}_{2}$, $\mathrm{C}_{3}$ et $\mathrm{C}_{13}$ ont été isolées dans des lésions de pneumonie enzootique. L'association avec des bactéries était constante: des staphylocoques et $P$. hemolytica pour les deux premières, une flore plus complexe (Pseudomonas, Alcaligenes, Achromobacter) pour la troisième; dans ce dernier cas, l'agent pathogène initial était le myxovirus parainfluenza III (*).

L'infection par la souche $\mathrm{C}_{14}$ était concomitante d'une infestation à Mullerius capillaris qui, comme on le sait, entretient par son action irritante des foyers de broncho-pneumonie chronique.

Il est donc vraisemblable que ces mycoplasmes n'ont qu'un rôle tout à fait secondaire d'infection associée; leur multiplication dans l'arbre respiratoire doit être favorisée par d'autres agents pathogènes: virus, bactéries, parasites.

Des exemples de tels complexes infectieux existent déjà en pathologie vétérinaire :

(*) Communication personnelle de notre confrère A. Provost. 
- L'infection à $M$. bovirhinis et à $M$. dispar, hôtes fréquents et bien supportés du tractus respiratoire des jeunes bovins, s'étend jusqu'au tissu pulmonaire, souvent de façon concurrente avec les bactéries du genre Pasteurella, lorsqu'un virus a créé les premières lésions.

- L'infection pulmonaire à $M$. hyorhinis chez le porc est toujours secondaire à l'intervention d'un virus (grippe, peste porcine) ou associée à la pneumonie enzootique due à $M$. hyopneumoniae.

- Chez la chèvre, un précédent existe avec $M$. arginini que nous avons eu l'occasion d'isoler déjà deux fois de lésions pulmonaires à flore bactériennel complexe.

Enfin, si on range ces souches dans l'espèce $M$. dispar, nous en discuterons plus loin, on a le droit de croire qu'elles ont, en pathologie caprine, un rôle identique à celui que l'espècetype joue en pathologie bovine.

3. D'ordre taxonomique: la parenté sérologique qui unit ces souches à $M$. dispar autorise-t-elle à les classer sous cette même étiquette ? Ou bien les petites différences observées grâce à la précipito-diffusion et à l'analyse électrophorétique permettent-elles d'en faire une espèce distincte ? Certes, le test très spécifique de l'inhibition du métabolisme n'a pas été exécuté et il est vraisemblable qu'il aurait été utile, encore qu'on ne voit pas bien a priori de quelle façon il serait un meilleur moyen de classification que l'analyse antigénique effectuée dans cette étude.
Nous avons déjà évoqué ce problème du choix des critères à propos d'une souche de mycoplasme rattachée à l'espèce $M$. capri [PERREAU (12)], pour laquelle il se posait de façon très semblable.

Aussi considérons-nous aujourd'hui, jusqu'à preuve du contraire, que ces souches caprines ne représentent qu'un variant de l'espèce dispar, décrite initialement chez les bovins; ne peut-on admettre sur un plan dogmatique qu'il existe un groupe dispar, tout comme il existe un groupe capri?

\section{CONCLUSION}

Les quatre souches de mycoplasmes étudiées ici, d'origine géographique très différente (Ethiopie, France, Tchad) mais toutes isolées de lésions pulmonaires des chèvres, doivent être rattachées à l'espèce $M$. dispar (GOURLAY et LEACH, 1970), dont elles ne sont probablement qu'une variété particulière à la chèvre.

\section{Remerciements}

Nos vifs remerciements vont à nos confrères qui, grâce à des prélèvements soigneux, nous ont permis de faire cette étude : M. Vigier, au laboratoire de Debré-Zeit (Ethiopie); F. Bertin, au laboratoire de Farcha (Tchad); Tram Cuong et $\mathrm{A}$. Vallée de l'Institut Pasteur à Paris.

\section{SUMMARY}

Goat mycoplasma closely related to Mycoplasma dispar species

Four mycoplasma strains isolated from goats affected by enzootic pneumonia or lungworm disease, originating from different geographic regions (Ethiopia, France, Chad), seem to belong to a same species, very closely related to $M$. dispar (Gourlay and Leach, 1970).

This conclusion follows from a comparative study about their cultural, metabolic and antigenic properties. The serological tests used here are: sero-agglutination, fluorescent antibody technique, gel immunoprecipitation, growth inhibition; the antigenic analysis has been completed by acrylamide gel electrophoresis.

\section{RESUMEN}

Micoplasmos de la cabra emparentados a la especie Mycoplasma dispar

Cuatro cepas de micoplasmos aisladas en la cabra a partir de lesiones de neumonia enzootica y de bronquitis verminosa, de origen geográfica muy diferente (Etiopia, Francia, Chad), pertenecen, a lo que parece, a una misma especia, muy cercana de $M$. dispar (Gourlay y Leach, 1970). 
Esta conclusión resulta de un estudio comparativo sobre sus caracteres en cultivos, sus caracteres metabólicos y antigenicos. Los métodos serologicos son la sero-aglutinación, la inmunofluorescencia, la precipitodifusión en medio gelificado, la inhibición de crecimiento; Se completa el análisis antigenico por la electroforesis de gel de poliacrilamida.

\section{BIBLIOGRAPHIE}

1. ALUOTTO (B. B.) et collab. Standardized bacteriologic techniques for the characterization of mycoplasma species. Int. J. syst. Bact., 1970, 20 (1) : $35-58$

2. CLYDE (W. A.). Mycoplasma species identification based upon growth inhibition by specific antisera. J. Immunol., 1964, 92 (6): 958-965.

3. COTTEW (G.S.). Diseases of sheep and goats caused by mycoplasmas, in: SHARP (J.T.), ed. The role of mycoplasmas and L-forms of bacteria in disease. Springfield, Ill., U.S.A., C. C. Thomas, 1970 , pp. $198-211$

4. COTTEW (G.S.) et LEACH (R.H.). Mycoplasmas of cattle, sheep and goats, in: HAYFLICK (L.), ed. The mycoplasmatales and the L-phase of bacteria. Amsterdam, North-Holland Publ. Co, 1970, pp. 527-570.

5. GOURLAY (R.N.) et LEACH (R. H.). A new mycoplasma species isolated from pneumonic lungs of calves ( Mycoplasma dispar » sp. nov.). J. med. Microbiol., 1970, 3 : 111-123.

6. HERS, cité par LEMCKE (R.M.). Media for mycoplasmataceae. Lab. Pract., 1965, $14: 712$.

7. HUDSON (J. R.), COTTEW (G.S.) et ADLER (H. E.). Diseases of goats caused by mycoplasmas a review of the subject with some new findings. Ann. N.Y. Acad. Sci., 1967, 143: 287.

8. LEACH (R. H.). The occurrence of Mycoplasma arginini in several animal hosts. Vet. Rec., 1970 , 87: 319-320.

9. MEYER (J. F.). Les maladies infectieuses respiratoires des petits ruminants. Thèse doctorat vétérinaire. Paris. 1970. No 87.

10. PERREAU (P.). Identification de certaines souches de mycoplasmes de la chèvre à l'espèce Mycoplasma mycoides var. mycoides. Rev. Elev. Méd. vét. Pays trop., 1971, 24 (3) : 343-348.

11. PERREAU (P.), GAYT (P.) et MONNIER (J.). La méthode d'immunofluorescence et l'identification des mycoplasmes. Rev. Elev. Méd. vét. Pays trop., 1969, 22 (4): 481-493.

12. PERREAU (P.), TRAM CUONG et VALLEE (A.). Isolement d'un mycoplasme du groupe Mycoplasma mycoides var. capri à partir d'un lait de mammite chez la chèvre. Bull. Acad. vét., 1972, 45 (3) : 109-116.

13. RAZIN (S.) et ROTTEM (S.). Identification of mycoplasma and other micro-organisms by polyacrylamide-gel electrophoresis of cell proteins. J. Bact., 1967, 94 (6) : 1807-1810. 